\title{
Optimization of ultrasound extraction condition of phospholipids from palm-pressed
}

fiber.

\begin{abstract}
A central composite design (CCD) was employed to study the effect of ultrasound-assisted extraction (UAE) conditions namely amplitude (10-90\%), cycle $(0.1-1.0 \mathrm{~W} / \mathrm{s})$ and sonication time (5-30 min) on the extraction yield of phospholipids (PL) from palm-pressed fiber (PPF). Overall extraction efficiency and individual extraction yield of phosphatidylethanolamine (PE) and phosphatidylcholine (PC) were considered as response variables. The significant ( $\mathrm{p}$ $<0.05)$ response surface models with high coefficients of determination values (R2) ranged from 0.873 to 0.984 were fitted for the experimental data, thus indicating a satisfactory adjustment of the polynomial response models for describing the extraction efficiencies of the PL. The combined level of amplitude (20\%), cycle $(0.2 \mathrm{~W} / \mathrm{s})$ and sonication time (30 min) was predicted to provide the highest PL extraction efficiency. Under the optimum condition, the response values obtained for overall extraction efficiency and individual extraction yield of PE and PC were $110(\mathrm{mg} / \mathrm{g}), 12570$ and $5426(\mathrm{mg} / \mathrm{kg})$, respectively.
\end{abstract}

Keyword: Central composite design; Ultrasound-assisted extraction; Amplitude; Cycle; Extraction time; Phospholipids; Palm-pressed fiber. 